\title{
Model predictive control of sea wave energy converters - Part I: a convex approach for the case of a single device
}

\author{
Guang Li ${ }^{\mathrm{a}}$, Mike Belmont ${ }^{\mathrm{b}}$ \\ ${ }^{a}$ Department of Mechanical and Nuclear Engineering, Pennsylvania State University, \\ State College, PA, 16802. \\ ${ }^{b}$ College of Engineering, Mathematics and Physical Sciences, University of Exeter, \\ Harrison Building, North Park Road, Exeter, EX4 4QF, UK.
}

\begin{abstract}
This paper investigates model predictive control (MPC) of a single sea wave energy converter(WEC). By using control schemes which constrain certain quantities, such as the maximum size of the feedback force, the energy storage for actuators and relative heave motion, it is possible for control to not only improve performance but to directly impact strongly on design and cost. Motivated by this fact, a novel objective function is adopted in the MPC design, which brings obvious benefits: First, the quadratic program (QP) derived from this objective function can be easily convexified, which facilitates the employment of existing efficient optimization algorithms. Second, this novel design can trade off the energy extraction, the energy consumed by the actuator and safe operation. Moreover, an alternative QP is also formulated with the input slew rate as optimization variable, so that the slew rate limit of an actuator can be explicitly incorporated into optimization. All these benefits promote the real-time application of MPC on a WEC and reduced cost of hardware.
\end{abstract}

Keywords: wave energy, model predictive control, quadratic programming

\section{Introduction}

A sea wave energy converter (WEC) is a device used to harvest sea wave energy. Extracting the maximum possible time average power from WECs,

Email addresses: gul15@psu.edu (Guang Li), M.R.Belmont@exeter.ac.uk (Mike Belmont) 
while reducing the risk of device damage and at the same time minimizing the device cost, involves a combination of good fundamental engineering design of a device and effective control of its operation. The linking of control and basic design is not the conventional approach because control schemes are typically overlaid upon existing designs. However, minimizing certain quantities such as feedback force, stored energy for actuators and the relative heave motion using some control strategy has very marked direct effects upon design/cost.

In this paper, we investigate the control aspect of WECs. In particular, we focus on a typical type of WECs, called point absorbers, whose dimensions are small compared with the wave length of incoming waves.

Various control methods have been explored to improve energy extraction, such as impedance matching by tuning the dynamical parameters of the devices [1-4], and latching control by locking the body at some moments to keep the velocity in phase with the excitation force [5-10]. There are also some optimization based control strategies developed for WECs. [5] uses Lagrange multipliers to determine the optimal velocity profile. In [11] an linear quadratic Gaussian (LQG) control problem is formulated, with the assumption that no constraints are present. However, all these control methods are only suboptimal. More recent works [12-17] show that maximizing energy extraction while maintaining the safe operation of WEC is essentially a constrained optimization problem and the concept of model predictive control (MPC), or receding horizon control (RHC), can be potentially employed as the WEC control strategy. [18] uses a MPC as a benchmark for comparative evaluation of the performance of a simple controller. MPC is an online optimization technique. It resolves an optimization problem at each sampling instant to yield an optimal control sequence, the first of which is applied to the plant as the control input. This online optimization feature requires a fast optimization algorithm, especially when it is applied to mechanical systems, e.g. [19]. Conventionally, the optimization is formulated as a convex quadratic programm (QP) [16], so that efficient optimization algorithms such as the interior point method and the active set method can be employed. However, the optimization associated with the WEC control may not be guaranteed to be convex as shown later in this paper. This problem impedes the implementation of these efficient algorithms. In this paper we show how to overcome this problem by adopting a novel cost function.

To present the point absorber control problem, we use the Power Buoy device PB150 developed by OPT Inc, see [20], as a concrete example, which is illustrated in Fig. 1 and is studied in [16]. On the sea surface is a float, 


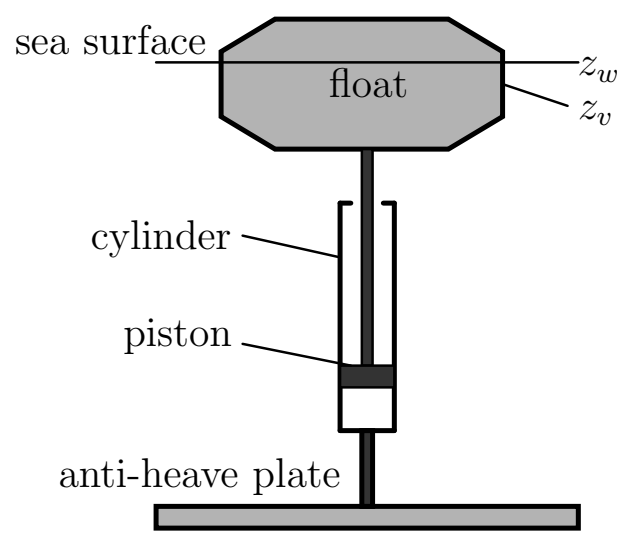

Figure 1: Schematic diagram of the point absorber

below which hydraulic cylinders are vertically installed. These cylinders are attached at the bottom to a large area anti-heave plate whose vertical motion is designed to be negligible compared to that of the float. The heave motion of the float drives the pistons inside the hydraulic cylinders to produce a liquid flow. The liquid drives hydraulic motors attached to a synchronous generator. From here, the power reaches the grid via back-to-back $\mathrm{AC} / \mathrm{DC} / \mathrm{AC}$ converters; see [21] for more details related to the power electronics. Here $z_{w}$ is the water level, $z_{v}$ is the height of the mid-point of the float. The control input is the $q$-axis current in the generator-side power converter, to control the electric torque of the generator [22]. The generator torque is proportional to the force $f_{u}$ acting on the pistons from the fluid in the cylinders. Since the motion of the float imposes a velocity $v=\dot{z}_{v}$ on the piston, the extracted power $P(t)$ at time $t$ is expressed as

$$
P=-f_{u} v
$$

Note that, different from [16], it is assumed here the directions of $f_{u}$ and $v$ are the same, so that a minus sign in (1) is needed. This is merely a matter of expression for the formulation of the QP. The extracted energy over a period $[0, T]$ is therefore

$$
-\int_{0}^{T} f_{u} v d t
$$

MPC aims to maximize the energy in its discrete time version, which amounts 
to minimize the cost function

$$
J=\sum_{k=0}^{N} f_{u}(k) v(k)
$$

where $f_{u}(k)$ and $v(k)$ are the discrete time values of $f_{u}(t)$ and $v(t)$ sampled with a sampling period $T_{s}$. To avoid damage, and for overall performance reasons, two constraints have to be considered in any real WEC. One concerns the relative motion of the float to the sea surface (it should neither sink nor raise above the water and then slam), which can be expressed as

$$
\left|z_{w}-z_{v}\right| \leq \Phi_{\max }
$$

Since $z_{w}-z_{v}$ is proportional to the buoyancy force $f_{s}$, (4) can be equivalently represented as

$$
\left|f_{s}\right| \leq z_{\max }
$$

The other constraint is on the control signal set by limitations on the allowable converter current. This constraint can be expressed as

$$
\left|f_{u}\right| \leq \gamma
$$

The control objective is to maximize the extracted energy subject to the constraints (5) and (6).

However, this constrained optimization problem leads to a non-convex QP, which prevents us from using efficient optimization algorithms to resolve it efficiently online. Some methods have been proposed to overcome this problem. In [23], the WEC control is formulated as a constrained optimization problem which is approximated by a resulting concave quadratic function. In [16], we aim to resolve this non-convex optimization problem directly. This constrained optimal control problem is analyzed using Pontryagin's minimum principle, and the analysis result facilitates the employment of dynamic programming (DP) for the online optimization. This is because the resulting control takes a bang-bang type of control, that is, only the maximum or minimum of the allowed control signal is used as input at each sampling instant. This allows the cost function to be evaluated only along two trajectories generated by the two boundary input values, so that the computational burden is significantly reduced. However, there are two drawbacks related to this control method. Firstly, although simulations show 
that the computational speed is fast enough to guarantee the real time implementation of DP for a second order model, the exponentially increased computational burden for a higher order model, namely "the curse of dimensionality of DP", can invalidate its application. Secondly, since the control input only takes the maximum and minimum values, this has two impacts on the actuator: on the one hand, the physical design of the actuator may not allow the switch between the two boundary values at a very high frequency; on the other hand, the energy consumed by the actuator can be very large.

An alternative approach is to use a modified objective function to approximate the original one (3). This modified objective function takes the form of

$$
J=\sum_{k=0}^{N} f_{u}(k) v(k+1)
$$

which contains one sampling instant delay from input to output. In $[13,14]$, similar approaches are used, and the QP resulting from this approximated objective function is assumed convex, which enables the application of the conventional MPC. It is noticed that, possibly by mistake, the power expression in the objective function in [14] does not involve the delay term. We acknowledge the efficacy of this approximation method for many cases. However, in this paper, we show by examples that the assumption on the QP's convexity associated with the modified cost function may not always hold for all the possible parameter selections. Moreover, we demonstrate by simulations that the MPC based on this modified cost function can cause significant loss of extracted energy for a WEC in some cases.

Motivated by the existing results, the present paper aims to propose an efficient MPC control strategy to directly optimize the energy output and control signal. The MPC employs the following objective function

$$
J=\sum_{k=0}^{N}\left[f_{u}(k) v(k)+r f_{u}^{2}(k)+q f_{s}^{2}(k)\right]
$$

with the weight $r>0$ and $q>0$. Here the weighted term $r f_{u}^{2}(k)$ represents the consumed energy of the input signal, and $q$ is used to penalize $f_{s}$. The adoption of this objective function has the following benefits:

(i) The QP can be guaranteed to be convexified when the weight $r$ is chosen bigger than a certain value, and this value can be easily calculated; 
(ii) The energy consumed by the actuator can be dramatically reduced at the expense of a moderate loss of extracted energy, and the balance between the consumed energy and extracted energy can be flexibly traded off by tuning the weight;

(iii) The control signal can be smoothed, so that the requirement for the actuator's response limit is less stringent.

(iv) The purpose of penalizing $f_{s}$ in the cost function is mainly for guaranteeing the feasibility of the optimization problem. Since $f_{s}$, as a component of state variable, must be within a limit for safety reason, it is possible to have such a scenario that there is no way for any possible input force within given allowable limits to constrain $f_{s}$ within its safety limit. If this happens, then the optimization problem cannot yield a solution and thus the optimization is infeasible. This is not supposed to happen during the implementation of online constrained optimal control. Large incoming waves are more likely to cause such an infeasibility problem. Thus the weight $q$ provides an extra degree of freedom for tuning, so that the constraint on the heave motion of the buoy can be satisfied for large incoming waves. Of course, this extra cost can lead to a reduction of energy output. This is exactly the flexibility of this novel cost function can achieve for a trade-off between the safe operation (or design limit) of the WEC and the energy output under different wave conditions.

Based on (8), we also formulate another QP with the input slew rate as the optimization variable, which makes it possible to additionally restrict the actuator's slew rate within a certain range. The limitation on some actuators's slew rate can be crucial for its safe operation, and therefore significantly affects the actuator's cost. All these efforts make the real-time application of an MPC controller more realistic.

The approach developed in this paper is based on the assumption that at each sampling instant the wave profile for a certain future period can be estimated by some wave prediction algorithms, e.g. deterministic sea wave prediction (DSWP) [24-33], as presented in [16]. However, the methodology behind it is still valid when other alternative wave prediction algorithms are employed, e.g. [17]. We do not conduct robustness and performance analysis of the proposed methods regarding the wave prediction accuracy and prediction horizon, as this can be completed in a similar way as [16]. 
Table 1: Nomenclature

\begin{tabular}{l|l}
\hline Notation & Description \\
\hline$M^{T}$ & transpose of matrix $M$ \\
$\lambda_{\min }(M)$ & minimum eigenvalue of the matrix $M$ \\
$M>0$ & $M$ is a positive definite matrix \\
$\operatorname{He}(M)$ & equals $M+M^{T}$ \\
$\preceq$ & term-by-term inequality for two vectors \\
$\hat{v}(k+i \mid k)$ & estimate of $v(k+i)$ based on the measurement up to time $k ;$ \\
& $v(k):=\hat{v}(k \mid k)$ for conciseness. \\
$V_{a}^{b}(\hat{v}, k)$ & {$\left[\hat{v}(k+a \mid k)^{T}, \cdots, \hat{v}(k+b \mid k)^{T}\right]^{T}$ with $a, b \in \mathbb{N}$ and $a<b ;$} \\
$\Delta u(k)$ & $\begin{array}{l}V_{a}^{b}(\hat{v}, k):=V_{a}^{b} \text { for conciseness. } \\
\text { slew rate of } u(k), \text { i.e. } \Delta u(k)=u(k)-u(k-1)\end{array}$ \\
\hline
\end{tabular}

The structure of this paper is as follows. In Section 2, the dynamic model of the point absorber is established. In Section 3, we present the WEC optimization problem in a standard format and the quadratic programme associated with the cost function (8) is formulated. Section 4 addresses the convexity problems associated with the cost functions (3) and (7), and justifies the necessity of including the extra weighted term $r f_{u}^{2}$ in the cost function (8); moreover, to make a direct comparison of the QP solutions related to the cost functions (3) and (7), the difference-convex optimization is introduced. In Section 5, the MPC framework for WEC control is presented together with the Kalman observer design. Simulation results are demonstrated in Section 6. Finally, the paper is concluded in Section 7.

The nomenclature and the notation for the WEC modelling are summarized in Table 1 and Table 2 respectively.

\section{Model setup}

There are many existing approaches for modelling a point absorber in literature. We assume that the device under investigation of this paper is designed so that the vertical movement of the anti-heave plate is negligible compared with that of the float. With this assumption, the cylinder below the float can be considered to be attached to the seabed. For a more thorough investigation of the modeling issues of point absorbers, see $[7,34,35]$. In this section, we adopt a similar modelling procedure as [14]. We assume the oscillation of the device relative to the wave elevation is within some 
Table 2: WEC modelling variables and parameters

\begin{tabular}{l|l}
\hline Notation & Description \\
\hline$m_{s}$ & mass of the float \\
$m_{a}$ & added mass \\
$m$ & sum of added mass and the float mass \\
$k$ & hydrodynamic stiffness of the float \\
$D$ & constant damping coefficient \\
$\hat{D}(j \omega)$ & frequency-dependant damping coefficient \\
$h_{r}$ & impulse response of $\hat{D}(j \omega)$ \\
$z_{w}$ & sea surface elevation \\
$z_{v}$ & float elevation \\
$f_{u}$ & force of the piston (control input) \\
$f_{s}$ & force associated with the stiffness \\
$f_{d}$ & force associated with damping \\
$\rho$ & water density \\
$S$ & cross sectional area of the float \\
$g$ & gravitational constant \\
\hline
\end{tabular}

reasonable limit that the linear theory is applicable, which makes it possible to use a linear model to describe the WEC [36]. However, different from [14] where wave elevation is assumed to be small enough to be negligible, here the wave elevation is explicitly incorporated into the modelling, since we are concerned about the constraint satisfaction in terms of the difference of the elevations of the wave and float.

The mathematic model for this WEC can be described by

$$
m_{s} \ddot{z}_{v}=-f_{s}-f_{r}-f_{f}+f_{u}
$$

Here the buoyancy force $f_{s}:=k\left(z_{w}-z_{v}\right)$, with the hydrostatic stiffness $k=\rho g S$; the mechanical force $f_{f}:=D_{f} \dot{z}_{v}$ due to friction and viscosity [36]; the force applied on the piston used as the control input, $f_{u}$; the radiation force is calculated by $f_{r}:=\int_{-\infty}^{t} h_{r}(\tau)\left[\dot{z}_{v}(t-\tau)-\dot{z}_{w}(t-\tau)\right] d \tau+m_{a} \ddot{z}_{v}$ with the convolution part $h_{r}$ computed by boundary element methods (e.g. [37], WAMIT [38]) or approximated using analytical solutions for specific float geometry $[39,40]$. If we represent the convolution term by $f_{d}$ and assume the Fourier transform of $h(t)$ is $\hat{D}(j \omega)$, then $\hat{D}(j \omega)$ has an equivalent state space realization $\left(A_{r}, B_{r}, C_{r}, D_{r}\right)$, so that $f_{d}$ can be equivalently represented 
as a state space model

$$
\begin{aligned}
\dot{x}_{r} & =A_{r} x_{r}+B_{r}\left(\dot{z}_{v}-\dot{z}_{w}\right) \\
f_{d} & =C_{r} x_{r}
\end{aligned}
$$

where $x_{r} \in \mathbb{R}^{n_{r}}$. Using these relations, we can express the ODE (9) by a state-space model

$$
\begin{aligned}
& \dot{x}=A_{c} x+B_{u c} u+B_{w c} w \\
& y=C_{c} x \\
& z=C_{z} x
\end{aligned}
$$

where $w:=\dot{z}_{w}, u:=f_{u}, y:=\dot{z}_{v}, z:=f_{s}$ and $x:=\left[f_{s}, \dot{z}_{v}, x_{r}\right]^{T}$,

$$
\begin{array}{rlrl}
A_{c} & =\left[\begin{array}{ccc}
0 & -k & 0 \\
\frac{1}{m} & -\frac{D_{f}}{m} & -\frac{C_{r}}{m} \\
0 & B_{r} & A_{r}
\end{array}\right] & B_{w c}=\left[\begin{array}{c}
k \\
0 \\
-B_{r}
\end{array}\right] & B_{u c}=\left[\begin{array}{c}
0 \\
\frac{1}{m} \\
0
\end{array}\right] \\
C_{c}=\left[\begin{array}{lll}
0 & 1 & 0_{1 \times n_{r}}
\end{array}\right] & C_{z}=\left[\begin{array}{lll}
1 & 0 & 0
\end{array}\right]
\end{array}
$$

with $m:=m_{s}+m_{a}$. In this model, $\hat{D}(j \omega)$ can be deemed as a damping coefficient which varies with frequency. The added mass $m_{a}$ is also frequency dependent, but this dependence is relatively weak, as shown in e.g. [41]. So here we only incorporate the dynamics of the damping coefficient into the state space model as shown in $[7,14,36]$; the case for the added mass can be followed in a similar way, if required.

If the convolution term $f_{d}$ is approximated by $f_{d}:=D\left(\dot{z}_{v}-\dot{z}_{w}\right)$, then the state space model is simplified to a second order model with state variable $x:=\left[f_{s}, \dot{z}_{v}\right]^{T}$, and

$$
\begin{aligned}
A_{c} & =\left[\begin{array}{cc}
0 & -k \\
\frac{1}{m} & -\frac{D+D_{f}}{m}
\end{array}\right] & B_{w c} & =\left[\begin{array}{c}
k \\
\frac{D}{m}
\end{array}\right] \\
C_{c} & =\left[\begin{array}{ll}
0 & 1
\end{array}\right] & C_{z} & =\left[\begin{array}{ll}
1 & 0
\end{array}\right]
\end{aligned}
$$

Note that normally system identification methods together with some numerical tools are required to derive $\hat{D}(j \omega)$ and even the dynamics of the whole WEC from experimental data. The model we derived here is mainly for the purpose of demonstration of the MPC strategies by numerical simulation, although experimental data are used to roughly estimate the model for $\hat{D}(j \omega)$, as shown in section 6 . 


\section{Quadratic programming formulation for the WEC optimal con- trol problem}

To develop MPC scheme, (11) needs to be discretized to a discrete time model

$$
\begin{aligned}
x(k+1) & =A x(k)+B_{u} u(k)+B_{w} w(k) \\
y(k) & =C x(k) \\
z(k) & =C_{z} x(k)
\end{aligned}
$$

Based on (12), the constrained optimization problem is

$$
\begin{array}{ll} 
& \min _{U_{0}^{N}} \sum_{k=0}^{N}\left[y(k) u(k)+r u^{2}(k)+q z^{2}(k)\right] \\
\text { s.t. } & |z(k)| \leq z_{\max } \text { for } k=0,1, \ldots, N \\
& |u(k)| \leq u_{\max } \text { for } k=0,1, \ldots, N \\
& |\Delta u(k)| \leq \Delta u_{\max } \text { for } k=0,1, \ldots, M
\end{array}
$$

where the state constraint (13b) and input constraint (13c) correspond to (5) and (6) respectively. (13d) represents the constraint on the input slew rate.

In order to resolve (13), it needs to be converted into a QP form. This is done next for two cases. We start with the case in section 3.1 when the input magnitudes are used as optimization variables; in this case, the input slew rate constraint cannot be incorporated. Then in section 3.2, we extend to the case when the input slew rates are used as the optimization variables so that these quantities can be incorporated.

\subsection{MPC with input magnitudes as optimization variables}

For the ease of presentation, the objective function (13a) is rewritten in an equivalent concise form

$$
J:=\left(U_{0}^{N}\right)^{T} Y_{0}^{N}+\left(U_{0}^{N}\right)^{T} R U_{0}^{N}+\left(Z_{0}^{N}\right)^{T} Q Z_{0}^{N}
$$

where $R=r \times I_{N+1}$ and $Q=q \times I_{N+1}$. Propagating the state equation (12a) and using (12b) through the control horizon $N$ gives

$\hat{y}(k+i \mid k)=C A^{i} y(k)+\sum_{j=0}^{i-1} C A^{i-j-1} B_{u} \hat{u}(k+j \mid k)+\sum_{j=0}^{i-1} C A^{i-j-1} B_{w} \hat{w}(k+j \mid k)$ 
where $\hat{y}(k+i \mid k)$ denotes the state $y(k+i)$ estimated at time $k$, and the same meaning holds for $\hat{w}(k+j \mid k)$. (15) can be written in a matrix form

$$
Y_{0}^{N}=\Lambda_{x} x+\Phi_{U} U_{0}^{N}+\Phi_{W} W_{0}^{N-1}
$$

with $\Lambda_{x} \in \mathbb{R}^{(N+1) \times n}, \Phi_{U} \in \mathbb{R}^{(N+1) \times(N+1)}, \Phi_{W} \in \mathbb{R}^{(N+1) \times(N+1)}$, and more explicitly

$$
\begin{gathered}
\Lambda_{x}=\left[\begin{array}{c}
C \\
C A \\
C A^{2} \\
\vdots \\
C A^{N}
\end{array}\right] \quad \Phi_{U}=\left[\begin{array}{ccccc}
0 & & & & \\
C B_{u} & 0 & & & \\
C A B_{u} & C B_{u} & 0 & & \\
\vdots & \vdots & \ddots & \ddots & \\
C A^{N-1} B_{u} & C A^{N-2} B_{u} & \cdots & C B_{u} & 0
\end{array}\right] \\
\Phi_{W}=\left[\begin{array}{cccc}
0 & & & \\
C B_{w} & C B_{w} & & \\
C B_{w} & \vdots & \ddots & \\
\vdots & C A^{N-2} B_{w} & \cdots & C B_{w}
\end{array}\right]
\end{gathered}
$$

In a similar way, we can derive

$$
Z_{0}^{N}=\Lambda_{x, z} x(k)+\Phi_{U, z} U_{0}^{N}+\Phi_{W, z} W_{0}^{N-1}
$$

where $\Lambda_{x, z}, \Phi_{U, z}$ and $\Phi_{W, z}$ take the same forms as (17) and (18), but with $C$ replaced by $C_{z}$. Here the time derivative of the wave elevation at the current instant $k$ is $w(k)$; we assume that at instant $k$, the future estimated values, $\hat{w}(k+1 \mid k), \cdots, \hat{w}(k+N-1 \mid k)$, are available. These future wave data can be derived by some form of deterministic sea wave prediction (DSWP) algorithm.

Substituting (16) into (14) leads to a quadratic form

$$
J=\frac{1}{2}\left(U_{0}^{N}\right)^{T} \mathcal{H}_{u} U_{0}^{N}+\mathcal{F}_{u}^{T} U_{0}^{N}
$$

where

$$
\begin{aligned}
\mathcal{H}_{u} & :=\Phi_{U}+\Phi_{U}^{T}+2 R+2 \Phi_{U, z}^{T} Q \Phi_{U, z} \\
\mathcal{F}_{u} & :=\left(\Lambda_{x}+2 \Phi_{U, z}^{T} Q \Lambda_{x, z}\right) x(k)+\left(\Phi_{W}+2 \Phi_{U, z}^{T} Q \Phi_{W, z}\right) W_{0}^{N-1}
\end{aligned}
$$


with $\mathcal{H}_{u} \in \mathbb{R}^{N \times N}$ and $\mathcal{F}_{u} \in \mathbb{R}^{N}$.

The control input constraint (13c) and the state constraint (13b) can be written in a componentwise inequality

$$
A_{u} U_{0}^{N} \preceq b_{u}
$$

where

$$
A_{u}=\left[\begin{array}{c}
I \\
-I \\
\Phi_{U, z} \\
-\Phi_{U, z}
\end{array}\right], \quad b_{u}=\left[\begin{array}{c}
U_{\max } \\
U_{\max } \\
Z_{\max }-\Lambda_{x, z} x(k)-\Phi_{W, z} W_{0}^{N-1} \\
Z_{\max }+\Lambda_{x, z} x(k)+\Phi_{W, z} W_{0}^{N-1}
\end{array}\right]
$$

with $U_{\max }=[\underbrace{1, \ldots, 1}_{N+1}]^{T} \times u_{\max }$ and $Z_{\max }=[\underbrace{1, \ldots, 1}_{N+1}]^{T} \times z_{\max }$.

In summary, the QP with the cost function (20) subject to the constraint (21) is

$$
\begin{aligned}
& \qquad U^{*}=\arg \min _{U_{0}^{k}} \frac{1}{2}\left(U_{0}^{N}\right)^{T} \mathcal{H}_{u} U_{0}^{N}+\mathcal{F}_{u}^{T} U_{0}^{N} \\
& \text { s.t. } A_{u} U_{0}^{N-1} \preceq b_{u}
\end{aligned}
$$

\section{2. $M P C$ with input changing rates as optimization variables}

If the actuator's slew rate needs to be limited, the input slew rate has to be used as optimization variable, which is defined as $\Delta \hat{u}(k+j \mid k)=\hat{u}(k+j \mid k)-$ $\hat{u}(k+j-1 \mid k)$. We assume $M$ control moves of $\Delta \hat{u}(k+j \mid k)$ with $j=1, \ldots, M$ and a prediction horizon of $N$ steps for the output, with $M \leq N$. Then we have the following relations

$$
\begin{gathered}
\hat{u}(k+i \mid k)=u(k-1)+\sum_{j=0}^{i} \Delta \hat{u}(k+j-1 \mid k) \text { with } i=0,1, \ldots, M, \\
\hat{u}(k+M \mid k)=\hat{u}(k+M+1 \mid k)=\cdots=\hat{u}(k+N \mid k) .
\end{gathered}
$$

Equations (23a) and (23b) can be expressed by a matrix equation as

$$
U_{0}^{N}=T_{u} u(k-1)+T_{\Delta U} \Delta U_{0}^{M-1}
$$


where

$$
T_{u}=\left[\begin{array}{c}
I \\
I \\
\vdots \\
I \\
\vdots \\
I
\end{array}\right] \quad T_{\Delta U}=\left[\begin{array}{cccc}
I & 0 & 0 & 0 \\
I & I & 0 & 0 \\
\vdots & \vdots & \ddots & \\
I & I & \cdots & I \\
\vdots & \vdots & \cdots & \vdots \\
I & I & \cdots & I
\end{array}\right]
$$

with $I \in \mathbb{R}^{n_{u}}, T_{u} \in \mathbb{R}^{(N+1) n_{u} \times n_{u}}$ and $T_{\Delta U} \in \mathbb{R}^{(N+1) n_{u} \times M n_{u}}$.

Substitution of (24) into (16), then into (14) leads to

$$
J=\frac{1}{2}\left(\Delta U_{0}^{M-1}\right)^{T} \mathcal{H}_{\Delta u} \Delta U_{0}^{M-1}+\left(\Delta U_{0}^{M-1}\right)^{T} \mathcal{F}_{\Delta u}
$$

where

$$
\begin{aligned}
\mathcal{H}_{\Delta u}= & T_{\Delta U}^{T}\left(\Phi_{U}+\Phi_{U}^{T}+2 R+2 \Phi_{U, z} Q \Phi_{U, z}\right) T_{\Delta U}, \\
\mathcal{F}_{\Delta u}= & T_{\Delta U}^{T}\left[\left(\Lambda_{x}+2 \Phi_{U, z}^{T} Q \Lambda_{x, z}\right) x(k)\right. \\
& +\left(\Phi_{U}+\Phi_{U}^{T}+2 R+2 \Phi_{U, z}^{T} Q \Phi_{U, z}\right) T_{u} u(k-1) \\
& \left.+\left(\Phi_{W}+2 \Phi_{U, z}^{T} Q \Phi_{W, z}\right) W_{0}^{N-1}\right],
\end{aligned}
$$

with $\mathcal{H}_{\Delta u} \in \mathbb{R}^{M n_{u} \times M n_{u}}$ and $\mathcal{F}_{\Delta u} \in \mathbb{R}^{M n_{u}}$.

The constraints on input slew rate, input magnitude and state can be written in a componentwise inequality

$$
A_{\Delta u} \Delta U_{0}^{M-1} \preceq b_{\Delta u}
$$

where

$$
A_{\Delta u}=\left[\begin{array}{c}
I_{N} \\
-I_{N} \\
T_{\Delta U} \\
-T_{\Delta U} \\
\Phi_{U, z} T_{\Delta U} \\
-\Phi_{U, z} T_{\Delta U}
\end{array}\right], \quad b_{\Delta u}=\left[\begin{array}{c}
\Delta U_{\max } \\
\Delta U_{\max } \\
U_{\max }-T_{u} u(k-1) \\
U_{\max }+T_{u} u(k-1) \\
Z_{\max }-\Lambda_{x, z} x(k)-\Phi_{U, z} T_{u} u(k-1)-\Phi_{W, z} W_{0}^{N-1} \\
Z_{\max }+\Lambda_{x, z} x(k)+\Phi_{U, z} T_{u} u(k-1)+\Phi_{W, z} W_{0}^{N-1}
\end{array}\right]
$$

and $\Delta U_{\max }=[\underbrace{1, \ldots, 1}_{M}]^{T} \times \Delta u_{\max }$. 
Hence the QP with input slew rate constraint can be expressed as solving a QP with the cost function (26) and subject to the constraint (27):

$$
\begin{aligned}
& \Delta U^{*}=\arg \min _{\Delta U_{0}^{M-1}} \frac{1}{2}\left(\Delta U_{0}^{M-1}\right)^{T} \mathcal{H}_{\Delta u} U_{0}^{M-1}+\mathcal{F}_{\Delta u}^{T} \Delta U_{0}^{M-1} \\
& \text { s.t. } A_{\Delta u} \Delta U_{0}^{M-1} \preceq b_{\Delta u}
\end{aligned}
$$

\section{The convexity of the QPs and difference-convex optimization}

In this section, we discuss some issues related to the convexity of the QPs associated with the cost functions (3) and (7). Then we introduce how to use the difference-convex (DC) optimization method to approximately resolve the non-convex QP associated with (3).

\subsection{The convexity of the $Q P$ formulations}

We use simple examples to justify the necessity of including the weighted term $r u^{2}(t)$ in the cost function (13a) and demonstrate that convexity of (7) is not always guaranteed.

When $q=0$ and $r=0$ in the cost function (13a), the Hessian matrices $\mathcal{H}_{u}$ in (22) and $\mathcal{H}_{\Delta u}$ in (28) degenerate to $\mathcal{H}_{u}=\Phi_{U}+\Phi_{U}^{T}$ and $\mathcal{H}_{\Delta u}=$ $T_{\Delta U}^{T}\left(\Phi_{U}+\Phi_{U}^{T}\right) T_{\Delta U}$ respectively. In a similar way, we can also derive the Hessian matrices of the QPs corresponding to the cost function (7). When $U_{1}^{N+1}$ is used as the optimization variable, the Hessian matrix is $\mathcal{H}_{a}=\bar{\Phi}_{U}+$ $\bar{\Phi}_{U}^{T}$; when $\Delta U_{1}^{N+1}$ is used as the optimization variable, the Hessian matrix is $\mathcal{H}_{b}=\bar{T}_{\Delta U}^{T}\left(\bar{\Phi}_{U}+\bar{\Phi}_{U}^{T}\right) \bar{T}_{\Delta U}$. Here

$$
\bar{\Phi}_{U}=\left[\begin{array}{cccc}
C B_{u} & & & \\
C A B_{u} & C B_{u} & & \\
\vdots & \vdots & \ddots & \\
C A^{N-1} B_{u} & C A^{N-2} B_{u} & \cdots & C B_{u}
\end{array}\right]
$$

and $\bar{\Phi}_{W}$ take the same form with $\bar{\Phi}_{U}$ but with $B_{u}$ replaced by $B_{w} ; \bar{T}_{\Delta U}$ is derived by deleting the last row of $T_{\Delta U}$.

In [14], the QPs corresponding to the cost function (7) are assumed convex, i.e. $\mathcal{H}_{a}>0$ and $\mathcal{H}_{b}>0$. However, we can show by simple examples that this claim does not always hold. Suppose the second order state space model, with the assumption $D_{f}=0$ without loss of generality, is discretized using 
the zero-order hold method with a sampling period of $T_{s}$ and $D_{f}=0$ is assumed without loss of generality. The matrices associated with this discrete time model are

$$
\begin{aligned}
A & :=I+T_{s} A_{c}=\left[\begin{array}{cc}
1 & -k T_{s} \\
T_{s} / m & 1-D T_{s} / m
\end{array}\right] \\
B_{u} & =T_{s} B_{c u}=\left[\begin{array}{c}
0 \\
T_{s} / m
\end{array}\right] \\
B_{w} & =T_{s} B_{c w}=\left[\begin{array}{c}
k T_{s} \\
D T_{s} / m
\end{array}\right] \\
C & =C_{c}=\left[\begin{array}{ll}
0 & 1
\end{array}\right]
\end{aligned}
$$

Suppose prediction horizons are $N=2, M=2$, then

$$
\begin{array}{rlrl}
T_{\Delta U} & =\left[\begin{array}{ll}
1 & 0 \\
1 & 1 \\
1 & 1
\end{array}\right] & \Phi_{U} & =\left[\begin{array}{ccc}
0 & 0 & 0 \\
C B_{u} & 0 & 0 \\
C A B_{u} & C B_{u} & 0
\end{array}\right] \\
\bar{T}_{\Delta U}=\left[\begin{array}{ll}
1 & 0 \\
1 & 1
\end{array}\right] & \bar{\Phi}_{U}=\left[\begin{array}{cc}
C B_{u} & 0 \\
C A B_{u} & C B_{u}
\end{array}\right]
\end{array}
$$

In this scenario, we can investigate the convexity of the QPs corresponding to the cost function (7) by checking the positive definiteness of their associated Hessian matrices. Examples 1 and 2 in Appendix demonstrate that the convexity of the QPs corresponding to the cost function (7) can only be guaranteed within a limited range of values for parameters, and the range for the case with input slew rates as optimization variables is bigger than that for the case with input magnitudes as optimization variables. This means the QP formulated when using slew rate as optimization variable is more likely to be convex.

Next, the convexity of the QPs corresponding to the cost function (3) can also be demonstrated by Examples 3 and 4 in Appendix, which show that the positive definiteness of the Hessian matrices $\mathcal{H}_{u}$ and $\mathcal{H}_{\Delta u}$ cannot be satisfied for any possible parameter values. This means that the corresponding nonconvex QPs cannot be resolved efficiently using optimization algorithms such as active set method and interior-point method. However, the inclusion of the extra term $r u^{2}(k)$ with $r>0$ in the cost functions (8) or (13a) makes it possible to convexify the QPs, since this is equivalent to adding positive diagonal entries to the QPs. Suppose the Hessian of the QP associated 
with the cost function (3), i.e. $\sum_{k=0}^{N} f_{u}(k) v(k)$, is $\mathcal{H}$. Then we can choose $r>-\lambda_{\min }(\mathcal{H}) I$ such that $\mathcal{H}_{p}:=\mathcal{H}+r I>0$. Here $\mathcal{H}_{p}$ is the Hessian of the convex QP corresponding to the cost function $\sum_{k=0}^{N}\left[f_{u}(k) v(k)+r f_{u}^{2}(k)\right]$.

Furthermore, note that the conditions for guaranteeing the positive definiteness of the Hessian matrices are necessary and sufficient conditions when the discrete time model has matrices (29)-(31) and the prediction horizon is $N=2$. These conditions become only necessary when $N>2$. Moreover, the conditions can also be changed when a different discretization method, e.g. first-order-hold sampling, is used. Nevertheless, the properties of the positive definiteness of these Hessian matrices do not change.

\subsection{Difference-convex (DC) optimization}

Based on the analysis in the last subsection, we can develop a method to find the suboptimal solution of the cost function (3). We can use DC as a benchmark optimization method to make a direct comparison of the QP solutions corresponding to the two cost functions (3) and (7) respectively.

The Hessian matrices of the QPs associated with the cost function (3), i.e. $\sum_{k=0}^{N} f_{u}(k) v(k)$ can be expressed as

$$
\begin{aligned}
U_{k}^{*} & =\arg \min _{U_{k}}\left[\frac{1}{2} U_{k}^{T} \mathcal{H} U_{k}+U_{k}^{T} \mathcal{F}\right] \\
& =\arg \min _{U_{k}}\left[\frac{1}{2} U_{k}^{T}\left(\mathcal{H}+\mathcal{H}_{e}\right) U_{k}+U_{k}^{T} \mathcal{F}-\frac{1}{2} U_{k}^{T} \mathcal{H}_{e} U_{k}\right]
\end{aligned}
$$

with $\mathcal{H}_{e}>-\lambda_{\min }(\mathcal{H}) I$. Here $\lambda_{\min }(\mathcal{H})$ denotes the minimum eigenvalue of the matrix $\mathcal{H}$. Since $\mathcal{H}$ is not positive definite, we have $-\lambda_{\min }(\mathcal{H})>0$, so that $\mathcal{H}_{e}>0$ and $\mathcal{H}_{p}:=\mathcal{H}+\mathcal{H}_{e}>0$. Define

$$
\begin{aligned}
f\left(U_{k}\right) & :=\frac{1}{2} U_{k}^{T} \mathcal{H}_{p} U_{k}+U_{k}^{T} \mathcal{F} \\
g\left(U_{k}\right) & :=\frac{1}{2} U_{k}^{T} \mathcal{H}_{e} U_{k}
\end{aligned}
$$

Then both $f\left(U_{k}\right)$ and $g\left(U_{k}\right)$ are convex. Alternatively, they can be defined as the convex QPs corresponding to the cost functions $\sum_{k=0}^{N}\left[f_{u}(k) v(k)+r f_{u}^{2}(k)\right.$ $\left.+q f_{s}^{2}(k)\right]$ and $\sum_{k=0}^{N}\left[r f_{u}^{2}(k)+q f_{s}^{2}(k)\right]$ respectively. Optimization of (35) is thus equivalent to minimizing the difference between the two convex functions, which is known as a difference-convex (DC) optimization problem [42]. The 
first order Taylor series expansion of $g\left(U_{k}\right)$ is

$$
\begin{aligned}
g\left(U_{k}\right) & =\frac{1}{2} U_{k}^{T} \mathcal{H}_{e} U_{k}=\frac{1}{2} U_{k-1}^{T} \mathcal{H}_{e} U_{k-1}+U_{k-1}^{T} \mathcal{H}_{e}\left(U_{k}-U_{k-1}\right)+O\left(U_{k}-U_{k-1}\right) \\
& =U_{k}^{T} \mathcal{H}_{e} U_{k-1}-\frac{1}{2} U_{k-1}^{T} \mathcal{H}_{e} U_{k-1}+O\left(U_{k}-U_{k-1}\right) \\
& =\bar{g}\left(U_{k}\right)-\frac{1}{2} U_{k-1}^{T} \mathcal{H}_{e} U_{k-1}+O\left(U_{k}-U_{k-1}\right)
\end{aligned}
$$

We approximate $(35)$ by replacing $g\left(U_{k}\right)$ in $(35)$ by $\bar{g}\left(U_{k}\right)$

$$
\begin{aligned}
U_{k}^{*} & =\arg \min _{U_{k}}\left[\frac{1}{2} U_{k}^{T} \mathcal{H}_{p} U_{k}+U_{k}^{T} \mathcal{F}-U_{k}^{T} \mathcal{H}_{e} U_{k-1}\right] \\
& =\arg \min _{U_{k}}\left[\frac{1}{2} U_{k}^{T} \mathcal{H}_{p} U_{k}+U_{k}^{T}\left(\mathcal{F}-\mathcal{H}_{e} U_{k-1}\right)\right]
\end{aligned}
$$

Then the suboptimal solution can be found by resolving the convex QP (39) iteratively. The algorithm is summarized in Algorithm 1.

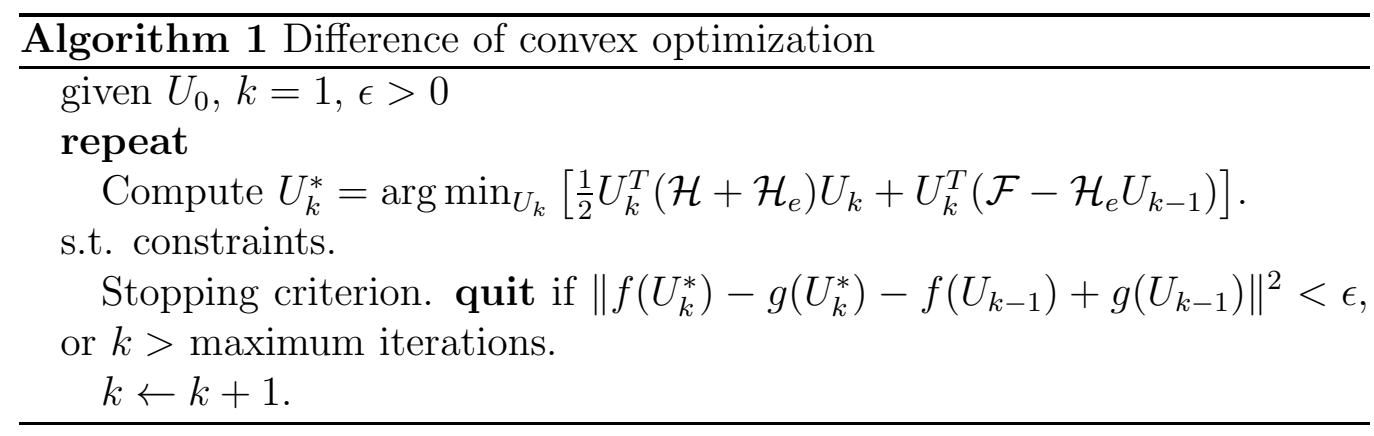

\section{MPC control framework}

The MPC WEC control framework is shown in Fig. 2. At the sampling instant $k$, the state $\hat{x}$ of the WEC's model is estimated by an observer $O(z)$, and if possible, the future wave's profile is predicted (using e.g. DSWP, [16]). With this information available, the evolution of the WEC's motion is predicted based on the WEC's dynamic model, over a certain fixed future period (called prediction horizon). This prediction can be formulated into a constrained optimization problem as demonstrated early in this paper. The 


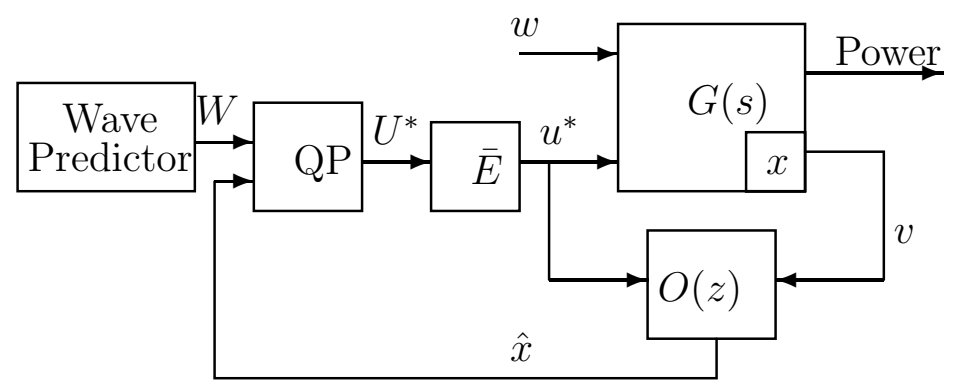

Figure 2: MPC WEC control framework

optimization algorithm is resolved online to yield a sequence of control input $U^{*}$. Only the first element of $U^{*}$, i.e. $u^{*}$ is used as the control input of the WEC. Note that if the optimization algorithm produces a sequence of control input changing signals, then a discrete time integration block $z /(z-1)$ is needed after the block $\bar{E}$ to derive $u^{*}$. At the next sampling instant $k+1$, the optimization is resolved again based on the new estimated state and predicted wave, and the whole process is repeated with the prediction horizon moving forward for one sampling period. For the details of the DSWP algorithm, refer to [24-33]. Here we briefly present the Kalman observer design for completeness, although Kalman filter is used for sea wave prediction [43, 44].

Consider a discrete time system

$$
\begin{aligned}
x(k+1) & =A x(k)+B_{u} u(k)+B_{w} w(k) \\
y(k) & =C x(k)+v(k)
\end{aligned}
$$

where $w(k)$ is the the time derivative of wave elevation, and $v(k)$ is the measurement noise. We suppose $w(k) \sim \mathcal{N}\left(0, R_{w}\right)$ and $v(k) \sim \mathcal{N}\left(0, R_{v}\right)$.

The estimator's equations are as follows

\section{Time Update:}

$$
\hat{x}(k+1 \mid k)=A \hat{x}(k \mid k)+B_{u} u(k)+B_{w} w(k)
$$

\section{Measured Updates:}

$$
\hat{x}(k \mid k)=\hat{x}(k \mid k-1)+L[y(k)-\hat{y}(k \mid k-1)]
$$

\section{Predicted Output Computation:}

$$
\hat{y}(k \mid k-1)=C \hat{x}(k \mid k-1)
$$


Then by substitutions, we have the state space representation of $O(z)$

$$
\begin{aligned}
\hat{x}(k+1 \mid k) & =A \hat{x}(k \mid k-1)+A L[y(k)-\hat{y}(k \mid k-1)]+B_{u} u(k)+B_{w} w(k) \\
& =A \hat{x}(k \mid k-1)+A L[y(k)-C \hat{x}(k \mid k-1)]+B_{u} u(k)+B_{w} w(k) \\
& =(A-L A C) \hat{x}(k \mid k-1)+B_{w} w(k)+B_{u} u(k)+L y(k)
\end{aligned}
$$

The Kalman gain $L$ is

$$
L=P C^{T}\left(C P C^{T}+R_{v}\right)^{-1}
$$

and the matrix $P$ is the solution of a discrete time algebraic Recatti equation:

$$
P=A P A^{T}-A P C^{T}\left(C P C^{T}+R_{v}\right)^{-1} C P A^{T}+B_{w} R_{w} B_{w}^{T}
$$

\section{Numerical simulation}

In this section, we present the simulation results using the MPC control strategies based on the cost functions (3), (7) and (8) respectively. For the ease of presentation, we refer to the MPC based on (3) as exact MPC (i.e. the MPC with the cost function exactly reflecting the extracted energy), the MPC based on (7) as approximated MPC, while the MPC based on (8) as the novel MPC. The model adopted contains a frequency dependent added damping term $\hat{D}(j \omega)$. Apart from damping, this model has similar dynamics to the 2nd order model in [16]. The stiffness is $k=6.39 \times 10^{5} \mathrm{~N} / \mathrm{m}$. The mass of the float is $m_{s}=1 \times 10^{4} \mathrm{~kg}$. The frequency independent added mass is $m_{a}=7 \times 10^{4} \mathrm{~kg}$. Then the total mass is $m=8 \times 10^{4} \mathrm{~kg} . D_{f}=0$ is assumed. The input magnitude constraint is $u_{\max }=3 \times 10^{5} \mathrm{~N}$ and the slew rate constraint is $\Delta u_{\max }=0.4 \times 10^{5} \mathrm{~N}$. The heave motion limit of the buoy is $\Phi_{\max }=1.2 \mathrm{~m}$. The frequency dependent added damping is

$$
\hat{D}(j \omega)=\frac{1.5 \times 10^{4} \times(j \omega+0.01)(j \omega+0.02)}{(j \omega+0.1)(j \omega+0.2)^{2}}
$$

This transfer function is estimated from real experimental data provided by OPT Inc. for the PB150 device. Its bode diagram is illustrated in Fig. 3. It shows that the maximum damping coefficient is $90 \mathrm{~dB}$ at $0.2 \mathrm{rad} / \mathrm{sec}$; this roughly matches the magnitude of the constant damping coefficient term $D=$ $2 \times 10^{5} \mathrm{Nm} / \mathrm{s}$ in the second order model used in [16]. The resulting 5th order 


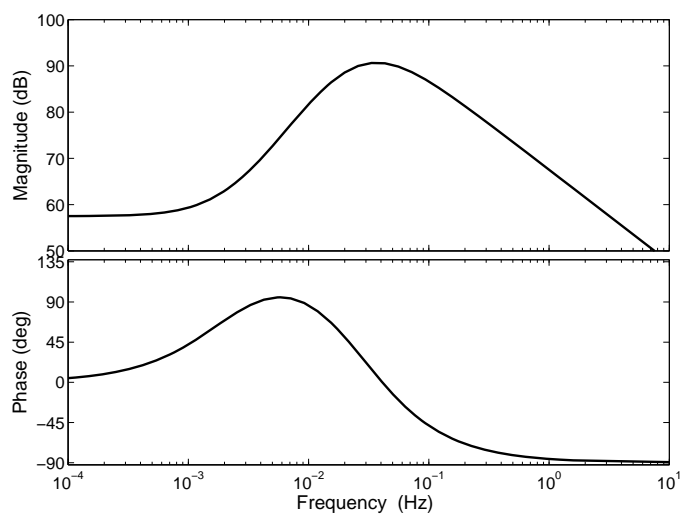

Figure 3: The bode diagram of the frequency-dependent damping coefficient.

continuous-time model rules out the implementation of the DP algorithm on computational ground. This model is discretized with a sampling rate $T_{s}=0.02 \mathrm{sec}$. Real sea wave data gathered off the coast of Cornwall, UK is used.

\subsection{Comparison between the exact $M P C$ and approximated $M P C$}

The wave heave magnitude and its derivative for a period of 50 seconds used for simulations are shown in Fig. 4. This period of wave data is relatively moderate compared to the wave date used in the next simulation. This is because the main purpose of this simulation is to show the energy loss brought by the approximation error of the approximated MPC, and therefore we do not want constraint violation to become the major issue, which will be demonstrated in the next simulation.

The input slew rate constrained is not incorporated into QP optimization and the optimization variable is the input magnitude sequence. The prediction horizon is $N=25$. The DC optimization is employed for the online optimization of the exact MPC. The weights for the Kalman observer design are chosen as $R_{w}=1$ and $R_{v}=1 \times 10^{-8}$. The input and state constraints are satisfied for both MPC control strategies. Fig. 5 shows that the energy output of the WEC controlled by approximated MPC is less than that of the exact MPC. This result is merely used to show roughly the approximated error brought by the one sampling instant delay in the cost function (7); it does not mean the exact MPC is a superior method used for WEC control. 


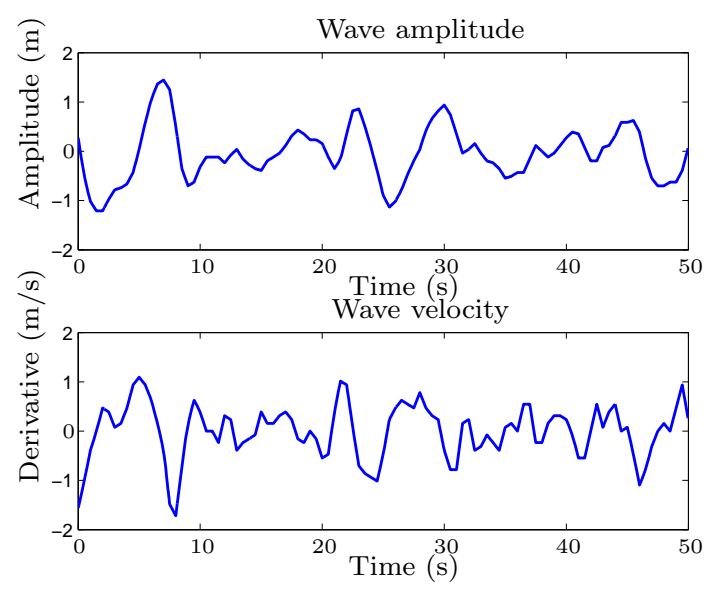

Figure 4: The wave amplitude and its derivative data used in simulations

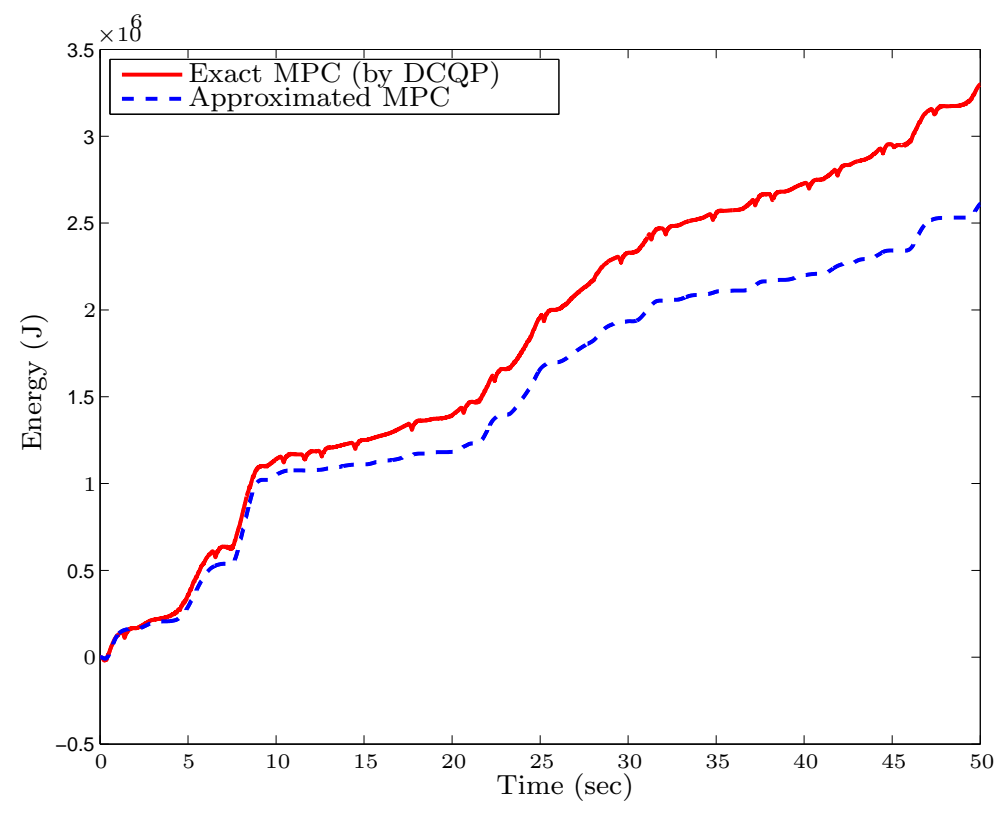

Figure 5: Extracted energy over time. We see that the MPC control with exact cost function generates more energy than the MPC control with approximated cost function. 


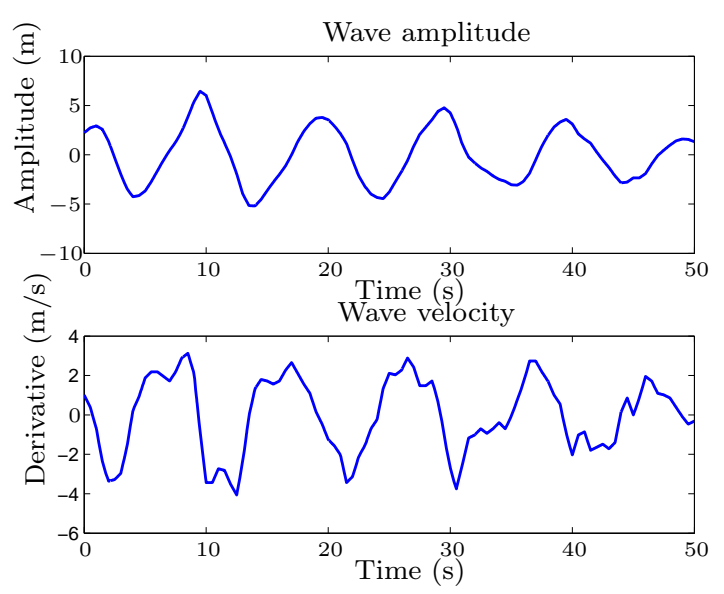

Figure 6: The wave amplitude and its derivative data used in simulations

\subsection{Comparison between the novel $M P C$, approximated $M P C$ and exact $M P C$}

The wave heave magnitude and its derivative for a period of 50 seconds used for simulations are shown in Fig. 6; the same data are also used in [16].

The sequence of input slew rates is used as the optimization variable and the input slew rate constraint is incorporated into QP formulation. Prediction horizons are $N=50$ and $M=30$. The weights for the Kalman observer design are chosen as $R_{w}=1$ and $R_{v}=1 \times 10^{-8}$. For the novel MPC, the weights in the cost function (8) are chosen as $r=3 \times 10^{-7}$ and $q=2 \times 10^{-7}$, which guarantees the positive definiteness of the Hessian matrix. It is noted that when the constraint on the relative heave motion is $[-1.2,1.2] \mathrm{m}$, the approximated MPC cannot always yield feasible solutions during simulations. For this reason, this limit is relaxed to $[-1.4,1.4] \mathrm{m}$ for the case of the approximated MPC simulations.

In the Figs. 7-11, the solid lines and dashed lines correspond to the simulation results from the novel MPC and the approximated MPC respectively.

Fig. 7 shows the heave motion trajectories: solid line is for the novel MPC and dashed line is for the approximated MPC. When the WEC is controlled by the proposed novel MPC, the relative heave motion constraint is satisfied for the whole period. But when the WEC is control by the approximated MPC, the constraint on relative heave motion is violated around 1.6, $10.3,12.3,13.5$ and 30.5 seconds. These constraint violations can potentially damage the WEC.

Fig. 8 shows the control inputs generated by the two control algorithms: 


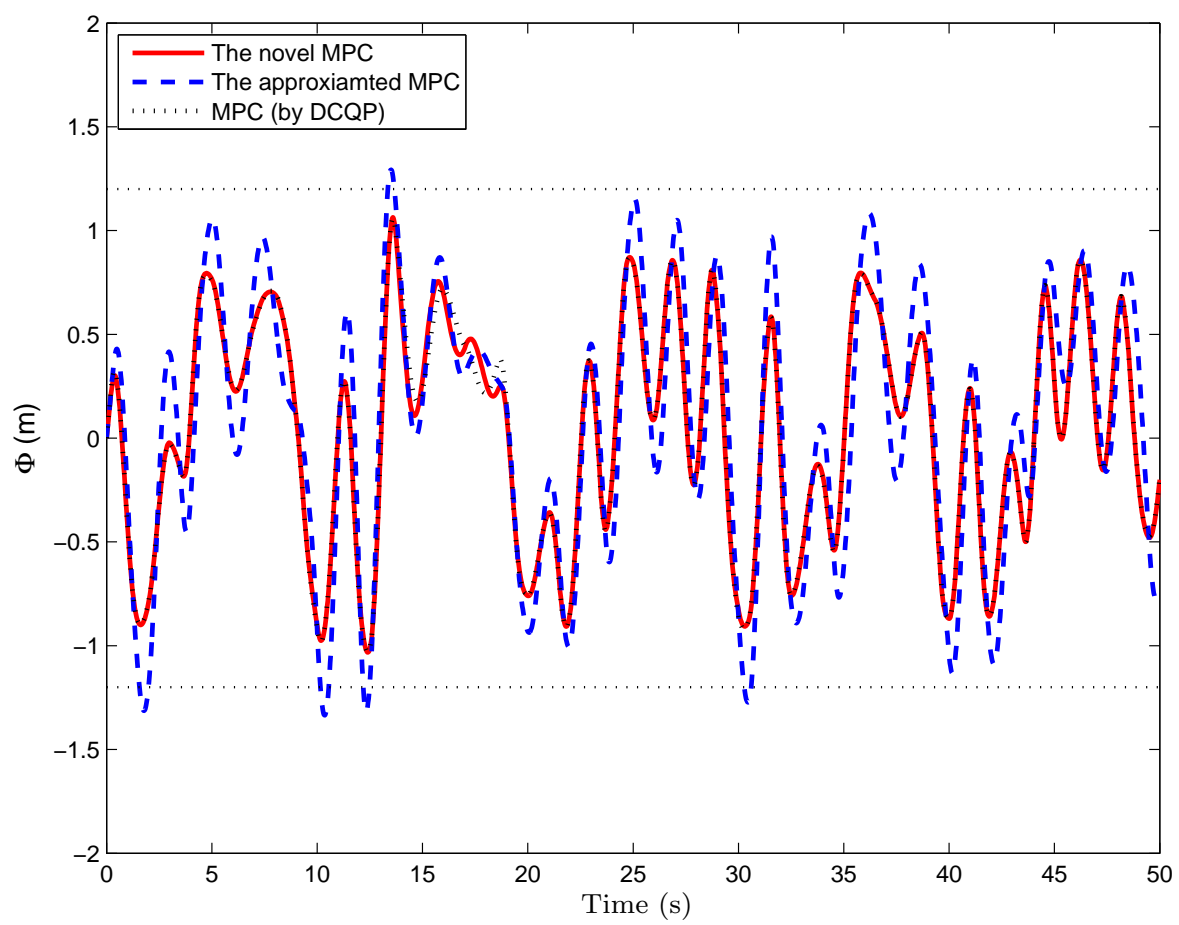

Figure 7: The vertical displacement difference between the water level and the mid-point of the float. The state constraint is satisfied for the whole period when the WEC is controlled by the novel MPC and exact MPC. But for the approximated MPC, constraint violations occur around 1.6, 10.3, 12.3, 13.5 and 30.5 seconds; this is not allowed due to the safety reason. 


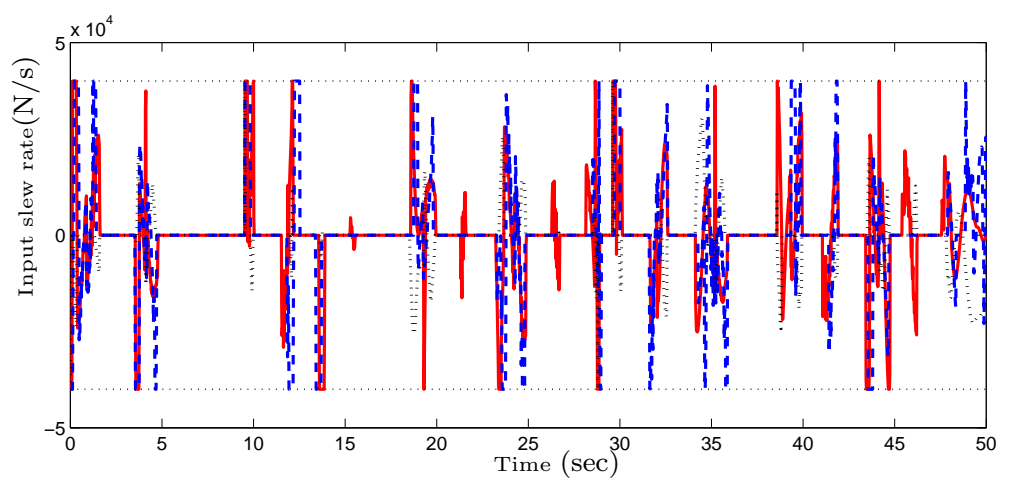

(a) Input slew rate

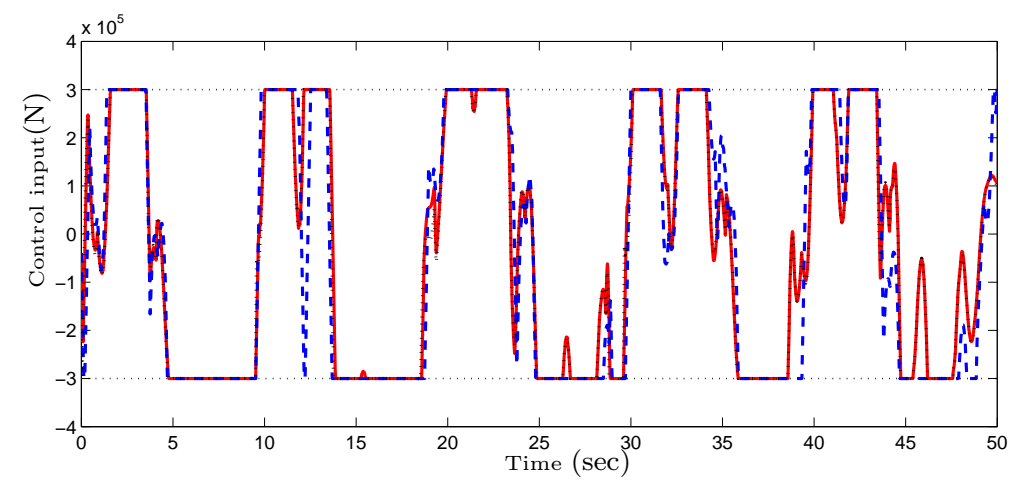

(b) Input magnitude

Figure 8: The control input magnitude and slew rate as a function of time. The magnitude and slew rate generated by both controllers satisfy the constraints. The solid line is for the MPC with the novel cost function (8). The dashed line is for the MPC with the approximated cost function (7), and the dotted line is for the exact MPC with DCQP as the optimization algorithm. 


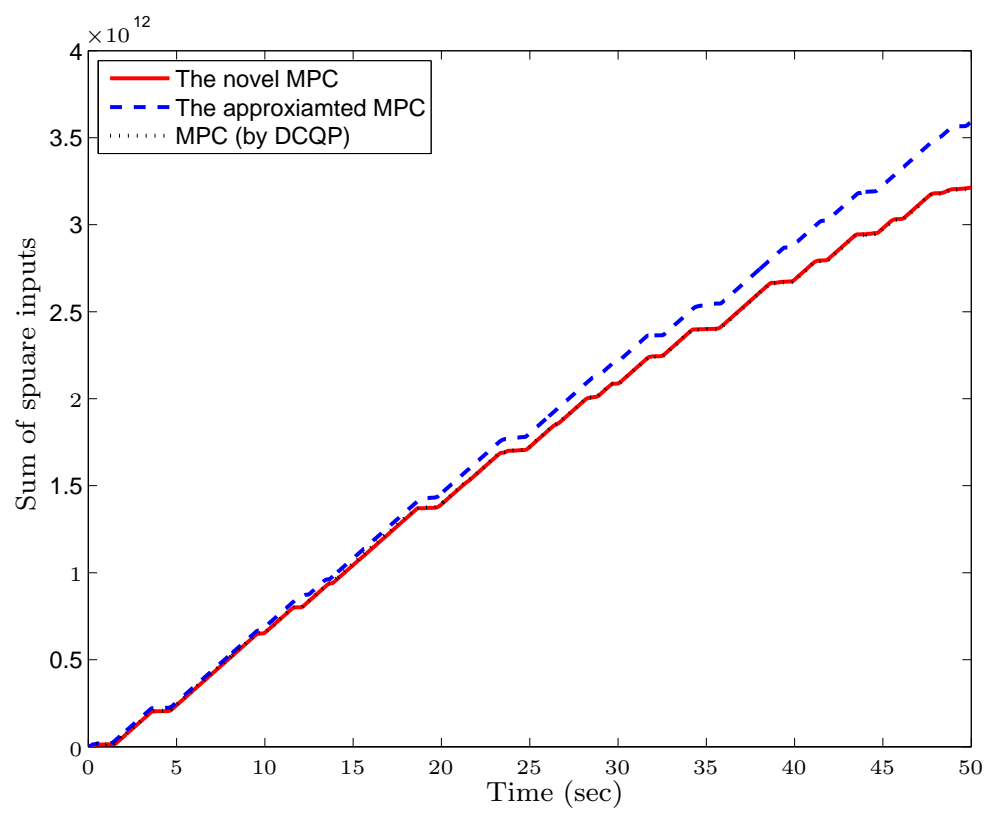

Figure 9: The sum of square of input over time; this term is proportional to the energy consumed by the actuator.

The input magnitude and input slew rate are both limited within the constraints. The benefit of imposing the slew rate constraint is reflected in the smooth change of the control signal provided to actuator, as shown in Fig. 8 (b), which would lead to a reduction of the actuator's cost. For example, just around 10 seconds, the input changes from $-3 \times 10^{5} \mathrm{~N}$ to $3 \times 10^{5} \mathrm{~N}$ gradually within 0.36 seconds, rather than jumping suddenly within one sampling period 0.02 second.

Fig. 9 shows the sum square of inputs (SSI) generated by each MPC controller. The SSI reflects the energy consumed by the actuator or the electricity needed to be provided to the actuator. This figure shows that the SSIs associated with the novel MPC and the exact MPC are indistinguishable and both slightly smaller than that of the approximated MPC. In fact, the magnitude of SSI can be tuned even smaller by increasing the value of $r$ in the cost function (8). Since the operation of the actuator needs electricity storage capacity to provide current, the smaller SSI also indicates the requirement of a smaller electricity storage device.

Fig. 10 and Fig. 11 show the power and energy generated by the three MPC WEC controllers respectively. The energy generated by the novel MPC 


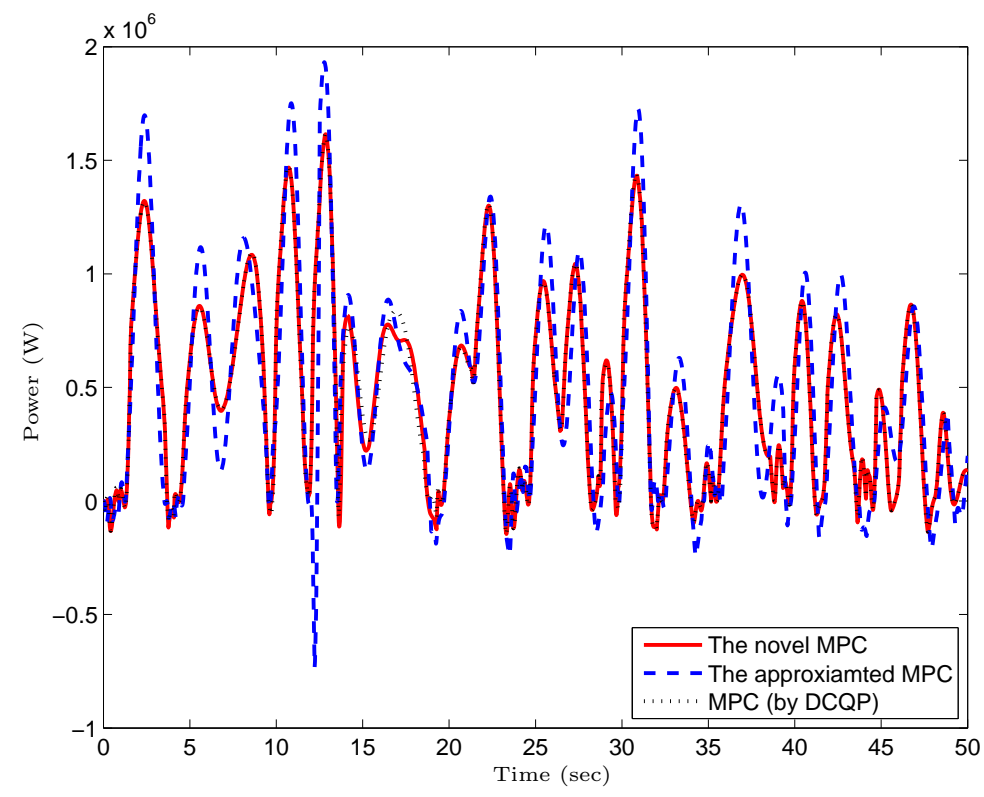

Figure 10: Extracted power as a function of time, over $50 \mathrm{sec}$.

and exact MPC are still indistinguishable and slightly less than that of the approximated MPC. This indicates two aspects: First, it should be noted that this relative smaller energy output is traded off by the smaller energy consumption by the actuator, and most importantly, the relative motion constraint is always satisfied for the whole simulation period compared with the constrained violation by the approximated MPC controller. In reality, this comparison is not even necessary because the approximate MPC violates the state constraints and so could not even be used under the real conditions. Second, the the extra terms involved in the novel MPC for penalizing the input variable and constrained state variable are nearly negligible compared with the case when only the output energy is used as the objective function.

\section{Conclusion}

We have proposed a novel MPC strategy for WEC control. This novel MPC can trade off the amount of energy output against the input energy consumption requirements of the actuator. It also explicitly penalizes the relative heave motion of the WEC, which guarantees feasible optimal solution and safe operation. The quadratic programme associated with this 


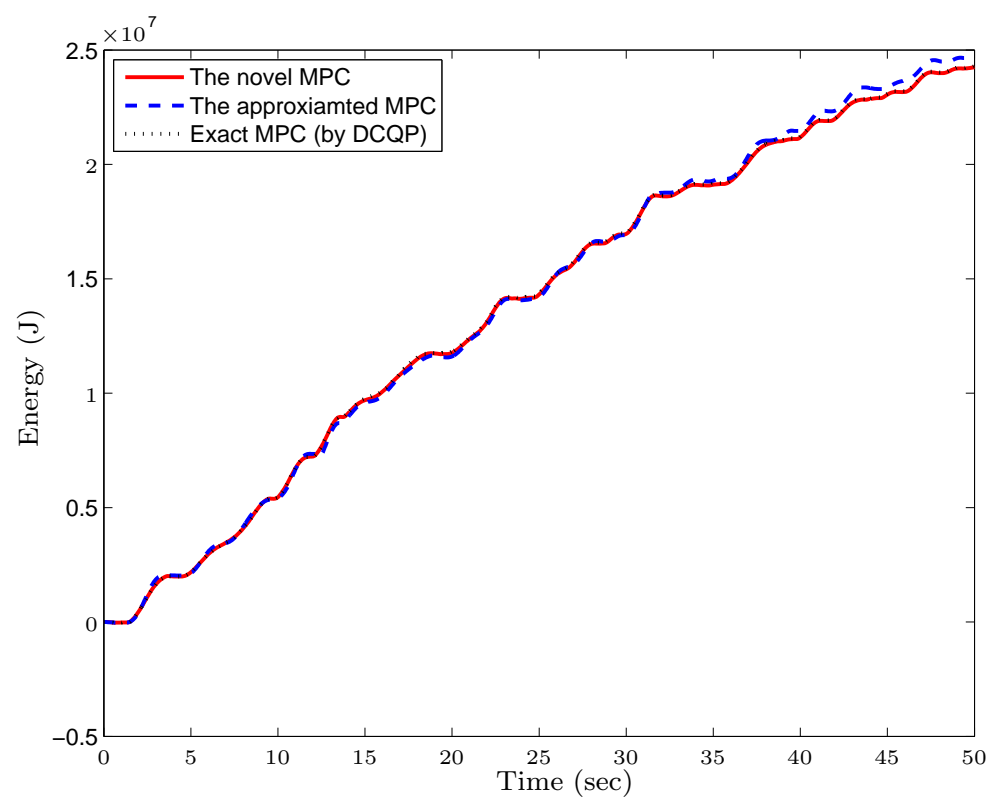

Figure 11: Extracted energy over time. The energy generated by the novel MPC is slightly less.

novel MPC can be tuned to be convex, which facilitates efficient online implementation. A typical type of point absorber is used as a study case. The simulation results confirm the efficacy of the proposed novel MPC strategy for WEC control.

\section{Bibliography}

[1] K. Budal, J. Falnes, Optimum operation of improved wave-power converter, Marine Science Communciations 3 (2) (1977) 133-150.

[2] K. Budal, J. Falnes, T. Hals, L. C. Iversen, T. Onshus, Model experiment with a phase controlled point absorber, in: Proc. of Second Int. Symp. on Wave and Tidal Energy, Cambridge, UK, 1981, pp. 191-206.

[3] K. Budal, J. Falnes, L. C. Iversen, P. Lillebekken, G. Oltedal, T. Hals, T. Onshus, A. Høy, The Norwegian wave-power buoy project, Tapir, Trondheim, 1982, pp. 323-344.

[4] G. A. Nolan, J. V. Ringwood, W. E. Leithead, S. Butler, Optimal damping profiles for a heaving buoy wave energy converter, in: The Fifteenth 
Int. Offshore and Polar Eng. Conf., Seoul, South Korea, 2005, pp. 477484.

[5] H. Eidsmoen, On theory and simulation of heaving buoy wave energy converters with control, Ph.D. thesis, Norwegian University of Science and Technology, Trondheim (1996).

[6] M. Greenhow, S. P. White, Optimal heave motion of some axisymmetric wave energy devices in sinusoidal waves, Applied Ocean Research 17 (1997) 141-159.

[7] J. Falnes, Ocean Waves and Oscillating Systems, Cambridge University Press, 2002.

[8] U. A. Korde, Latching control of deep water wave energy devices using an active reference 29 (11) (2002) 1343-1355.

[9] A. Babarit, A. H. Clément, Optimal latching control of a wave energy device in regular and irregular waves, Applied Ocean Research 28 (2006) 79-91.

[10] F. Fusco, J. V. Ringwood, Linear models for short-term wave forecasting, in: Proc. World Renewable Energy Conference, Glasgow, UK, 2008.

[11] J. Scruggs, S. Lattanzio, Optimal causal control of an ocean wave energy converter in stochastic waves, in: The European Wave and Tidal Energy Conference (EWTEC), Southampton, UK, 2011.

[12] J. Cretel, A. W. Lewis, G. Lightbody, G. P. Thomas, An application of model predictive control to a wave energy point absorber, in: IFAC Conference on Control Methodologies and Technology for Energy Efficiency, Vilamoura, Portugal, 2010.

[13] J. A. M. Cretel, G. Lightbody, G. P. Thomas, A. W. Lewis, Maximisation of energy capture by a wave-energy point absorber using model predictive control, in: Proc. 18th IFAC World Congress, Milano, 2011.

[14] J. Hals, J. Falnes, T. Moan, Constrained optimal control of a heaving buoy wave-energy converter, J. of Offshore Mechanics and Arctic Engineering 133 (1) (2011) 011401. 
[15] T. K. A. Brekken, On model predictive control for a point absorber wave energy converter, in: PowerTech, 2011 IEEE Trondheim, 2011, pp. 1-8.

[16] G. Li, G. Weiss, M. Mueller, S. Townley, M. B. Belmont, Wave energy converter control by wave prediction and dynamic programming, Renewable Energy 48 (2012) 392-403.

[17] F. Fusco, J. Ringwood, A study of the prediction requirements in realtime control of wave energy converters, Sustainable Energy, IEEE Transactions on 3 (1) (2012) 176-184.

[18] F. Fusco, J. Ringwood, A simple and effective real-time controller for wave energy converters, Sustainable Energy, IEEE Transactions on 4 (1) (2013) 21-30.

[19] G. Li, D. P. Stoten, J. Tu, Model predictive control of dynamically substructured systems with application to a servohydraulically actuated mechanical plant, IET Control Theory and Applications 4 (2) (2010) 253-264.

[20] Ocean Power Technologies, Inc., PB150 PowerBuoy, http://www.oceanpowertech.com/pb150.htm (2011).

[21] G. Weiss, G. Li, M. Mueller, S. Townley, , M. R. Belmont, Optimal control of wave energy converters using deterministic sea wave prediction, in: The Energy \& Materials Research Conference, Torremolinos, Malaga, Spain, 2012.

[22] Q.-C. Zhong, G. Weiss, Synchronverters: Inverters that mimic synchronous generators, IEEE Trans. on Industrial Electronics 58 (2011) 1259-1267.

[23] G. Bacelli, J. V. Ringwood, J.-C. Gilloteaux, A control system for a selfreacting point absorber wave energy converter subject to constraints, in: the 18th IFAC World Congress, Milano, Italy, 2011.

[24] E.L. Morris and H.K. Zienkiewicz and M.M.A. Purzanjani and J.O. Flower and M.R. Belmont, Techniques for sea state prediction, in: 2nd. Int. Conf. on Maneuvering and Control of Marine Craft, Southampton, 1992, pp. 547-571. 
[25] E. L. Morris, H. K. Zienkiewicz, M. R. Belmont, Short term forecasting of the sea surface shape, Int. J. Shipbld. Prog. 45 (1998) 381-400.

[26] M. R. Belmont, J. M. K. Horwood, J. Baker, Avoidance of phase shift errors in short term deterministic sea wave prediction, Journal of Marine Engineering and Technology (3) (2003) 21-26.

[27] M. R. Belmont, J. M. K. Horwood, R. W. F. Thurley, J. Baker, Filters for linear sea-wave prediction, Ocean Engineering 33 (2006) 2332-2351.

[28] M. R. Belmont, J. M. K. Horwood, The effect of frequency distribution in simulations of deterministic sea wave prediction, Int. Shipbld. Prog. (46) (1998) 265-276.

[29] M. R. Belmont, E. L. Morris, Adaptive measurement and signal processing strategies associated with deterministic sea wave prediction, Cambridge, UK, 1994, pp. 21-26.

[30] D. R. Edgar, J. M. K. Horwood, R. Thurley, M. R. Belmont, The effects of parameters on the maximum prediction time possible in short term forecasting of the sea surface shape, Int. Shipbuild. Progr. 47 (251) (2000) 287-301.

[31] L. Abusedra, M. R. Belmont, Prediction diagrams for deterministic sea wave prediction and the introduction of the data extension prediction method, Int. J. Sipbuilding Prog. 48 (1) (2011) 59-81.

[32] P. Naaijen, R. Huijsmans, Real time wave forecasting for real time ship motion predictions, in: 27th International Conference on Offshore Mechanics and Arctic Engineering (OMAE), Portugal, 2008, pp. 377-387.

[33] P. Naaijen, R. R. T. van Dijk, R. H. M. Huijsmans, A. A. El-Mouhandiz, Real time estimation of ship motions in short crested seas, ASME, 2009, pp. 243-255.

[34] A. Wacher, K. Nielsen, Mathematical and numerical modeling of the aquabuoy wave energy converter, Mathematics-in-Industry Case Studies 2 (2010) 16-33.

[35] A. A. E. Price, New Perspectives on Wave Energy Converter Control, Ph.D. thesis, The University of Edinburgh, Edingburgh, UK (2009). 
[36] J. Falnes, Optimum control of oscillation of wave-energy converters, International Journal of Offshore and Polar Engineering 12 (2) (2002) $147-155$.

[37] J. N. Newman, Marine Hydrodynamics, The MIT Press, Cambridge, MA, 1977.

[38] WAMIT, WAMIT, User manual, htt://wamit.com (2006).

[39] Waves due to a floating sphere making periodic heaving oscillations, Vol. 231, Proceedings of Royal Society, A, London, 1955.

[40] A. Hulme, The wave forces acting on a floating hemisphere undergoing forced periodic oscillations, Journal Fluid Mech. 121.

[41] J. Journèe, J. Pinkster, Introduction in ship hydromechanics, Lecture MT519, Delft University of Technology, draft Edition (April 2002).

[42] P. D. Tao, L. T. H. An, A dc optimization algorithm for solving the trust-region subproblem, SIAM Journal on Optimization 8 (2) (1998) 476-505.

[43] J. Hals, Modelling and phase control of wave-energy converters, Ph.D. thesis, Ph. D. dissertation, Department of Marine Technology, Norwegian University of Science and Technology, Trondheim (2010).

[44] F. Fusco, J. V. Ringwood, Short-term wave forecasting with AR models in real-time optimal control of wave energy, in: Proc. IEEE Intern. Symp. on Industrial Electronics, Bari, Italy, 2010, pp. 2475-2480.

\section{Appendix A. Examples for the convexity of QPs in Section 4}

\section{Example 1:}

For $N=2$, the Hessian matrix $\mathcal{H}_{a}$ is

$$
\begin{aligned}
\mathcal{H}_{a} & =\operatorname{He}\left(\bar{\Phi}_{U}\right)=\operatorname{He}\left(\left[\begin{array}{cc}
C B_{u} & 0 \\
C A B_{u} & C B_{u}
\end{array}\right]\right) \\
& =\left[\begin{array}{cc}
2 T_{s} / m & T_{s} / m\left(1-D T_{s} / m\right) \\
T_{s} / m\left(1-D T_{s} / m\right) & 2 T_{s} / m
\end{array}\right]
\end{aligned}
$$


Since $T_{s} / m>0$, we have $\mathcal{H}_{a}>0$ if and only if

$$
4-\left(1-D T_{s} / m\right)^{2}>0
$$

This inequality is satisfied if and only if $-1<D T_{s} / m<3$. Since $D>0$, $T_{s}>0$ and $m>0$, this condition can be replaced by $0<D T_{s} / m<3$.

\section{Example 2:}

For $N=2, M=2$, the Hessian matrix $\mathcal{H}_{b}$ is

$$
\begin{aligned}
\mathcal{H}_{b} & =\operatorname{He}\left(\bar{T}_{\Delta U}^{T} \bar{\Phi}_{U} \bar{T}_{\Delta U}\right)=\operatorname{He}\left(\left[\begin{array}{cc}
C A B_{u}+2 C B_{u} & C B_{u} \\
C A B_{u}+C B_{u} & C B_{u}
\end{array}\right]\right) \\
& =\operatorname{He}\left(\left[\begin{array}{ll}
T_{s} / m\left(1-D T_{s} / m\right)+4 T_{s} / m & 2 T_{s} / m \\
T_{s} / m\left(1-D T_{s} / m\right)+2 T_{s} / m & 2 T_{s} / m
\end{array}\right]\right)
\end{aligned}
$$

$\mathcal{H}_{b}>0$ if and only if

$$
\left[\begin{array}{cc}
2\left(5-D T_{s} / m\right) & 5-D T_{s} / m \\
5-D T_{s} / m & 4
\end{array}\right]>0
$$

By simple manipulations and considering $D T_{s} / m>0$, we see that (A.3) is satisfied if and only if $0<D T_{s} / m<5$.

Example 3: If $N=2$, then the Hessian matrix $\mathcal{H}_{u}$ is

$$
\mathcal{H}_{u}=\operatorname{He}\left(\Phi_{U}\right)=\left[\begin{array}{ccc}
0 & \left(C B_{u}\right)^{T} & \left(C A B_{u}\right)^{T} \\
C B_{u} & 0 & \left(C B_{u}\right)^{T} \\
C A B_{u} & C B_{u} & 0
\end{array}\right]
$$

Clearly, $\mathcal{H}_{u}$ is not positive definite due to the zero diagonal.

\section{Example 4:}

If $N=2, M=2$, then the Hessian matrix $\mathcal{H}_{\Delta u}$ is

$$
\begin{aligned}
\mathcal{H}_{\Delta u} & =\operatorname{He}\left(T_{\Delta U}^{T} \Phi_{U} T_{\Delta U}\right)=\operatorname{He}\left(\left[\begin{array}{ll}
C A B_{u}+2 C B_{u} & C B_{u} \\
C A B_{u}+2 C B_{u} & C B_{u}
\end{array}\right]\right) \\
& =\operatorname{He}\left(\left[\begin{array}{ll}
T_{s} / m\left(1-D T_{s} / m\right)+4 T_{s} / m & 2 T_{s} / m \\
T_{s} / m\left(1-D T_{s} / m\right)+4 T_{s} / m & 2 T_{s} / m
\end{array}\right]\right)
\end{aligned}
$$

$\mathcal{H}_{\Delta u}>0$ if and only if

$$
\left[\begin{array}{cc}
2\left(5-D T_{s} / m\right) & 7-D T_{s} / m \\
7-D T_{s} / m & 4
\end{array}\right]>0
$$

(A.6) is satisfied if and only if $D T_{s} / m<5$ and $\left(D T_{s} / m-3\right)^{2}<0$, which do not hold. 